\title{
Regulation of virulence factors by quorum sensing in Vibrio harveyi.
}

\begin{abstract}
Vibrio harveyi is an important aquatic pathogen that produces several virulence factors. In this study, the effect of quorum sensing, bacterial cell-to-cell communication, on the production of the virulence factors caseinase, gelatinase, lipase, hemolysin, and phospholipase, was investigated. The activity of virulence factors was studied through enzymatic plate assays using V. harveyi wild type and mutants with constitutively maximal or minimal quorum sensing activity. The results showed that quorum sensing negatively regulates phospholipase activity as higher activity was observed in mutants with minimal quorum sensing activity than in the mutant with maximal quorum sensing activity. Reverse transcriptase real-time PCR with specific primers revealed that the expression level of three phospholipase genes was 2-fold lower in the mutant with minimal quorum sensing activity than in the mutant with maximal quorum sensing activity. As far as we know, this is the first report of quorum sensing regulation of phospholipase. Finally, caseinase and gelatinase activity were positively regulated by quorum sensing, which is consistent with previous reports, and lipase and hemolysin activity were found to be independent of quorum sensing. Hence, the regulation is different for different virulence factors, with some being either positively or negatively regulated, and others being independent of quorum sensing. This might reflect the need to produce the different virulence factors at different stages during infection.
\end{abstract}

Keyword: Quorum sensing; Virulence factors; Phospholipase; Vibriosis; Vibrio harveyi. 